\title{
Mobility Trends and Effects on the COVID-19 Epidemic - Hong Kong, China
}

\author{
Rongna Zhang'; Zuoru Liang'; Mingfan Pang'; Xinping Yang'; \\ Jiewen W $\mathbf{u}^{1}$; Yuansheng Fang ${ }^{1}$; Hanran $\mathrm{Ji}^{1}$; Xiaopeng $\mathrm{Qi}^{1, \ldots+}$
}

\section{Summary \\ What is already known about this topic? \\ Several outbreaks of coronavirus disease 2019 (COVID-19) occurred in Hong Kong in 2020, and the response had varied results based on the strength of policy measures and on compliance of the population. \\ What is added by this report? \\ By analyzing data of COVID-19 cases in Hong Kong, combined with the Google Mobility Trends and Oxford COVID-19 Government Response Tracker, we make recommendations for the future prevention and control of the epidemic in Hong Kong. \\ What are the implications for public health practice? \\ Monitoring data reflecting multiple aspects, such as the epidemic situation, the mobility behavior of people, and government policy, is helpful for public health practitioners and policymakers to understand the interaction between various factors and to precisely adjust COVID-19 control policies.}

Hong Kong is a major port city that lies to the south of Shenzhen City of the mainland of China and acts as an important hub for international exchange. Hong Kong has a high population density and has difficulty controlling respiratory infectious diseases as a result. Though the medical standards in Hong Kong are comparatively good, the fluctuations in the coronavirus disease 2019 (COVID-19) epidemic, which peaked in mid-December 2020 (1), in Hong Kong placed great pressure on the hospital capacity as mobile cabin hospitals had to be used to supplement the situation (2). Accordingly, the testing capacity of Hong Kong has increased, and a peak was reached in September and is still expected to increase (3). Strict policies have helped control the epidemic but are accompanied by socioeconomic consequences, so the government response, community mobility, and daily confirmed cases were analyzed to determine how these policies and resulting community mobility were correlated with new cases.
Daily confirmed cases were extracted from February 15, 2020 to January 20, 2021 (1). Community mobility was extracted from Google Mobility Trends from February 15, 2020 to January 20, 2021. Google Mobility Trends records changes in the mobility of residents visiting the following six categories when compared to baseline: supermarket and pharmacy (including supermarkets, food warehouses, farmers markets, specialty food shops, and pharmacies); residential; workplaces; parks (national parks, public beaches, marinas, dog parks, plazas, and public gardens); public transport (transport hubs such as the subway/metro trains, buses, and other train stations); and retail and recreation (restaurants, cafes, shopping centers, theme parks, museums, libraries, and cinemas). Baseline was set as the mean value of mobility from January 3 to February 6, 2020, and location data for users were sampled every day to obtain percentage change from baseline. Higher mobility changes for the residential category indicated that citizens left home less often, which was aligned with epidemic control measures (4).

Government response was measured using the Oxford COVID-19 Government Response Tracker (OxCGRT) from February 15, 2020 to January 20, 2021 from Oxford University. The tracker used 19 indicators, of which the following 9 (called Stringency Index) were used in this study: school closing, cancel public events, restrictions on gatherings, close public transport, public information campaigns, restrictions on internal movement, workplace closing, stay at home requirements, and international travel controls. The index was measured on a scale of $0-100$, and a higher value indicated stricter control (5).

The number of daily new cases, the OxCGRT index and the 7-day moving average value of changes in mobility according to Google are shown in Figure 1. The epidemic in Hong Kong has experienced several fluctuations. The policy adjustment is lagging behind the change of daily new cases, and the longer the lag time, the more difficult the epidemic control will be. 

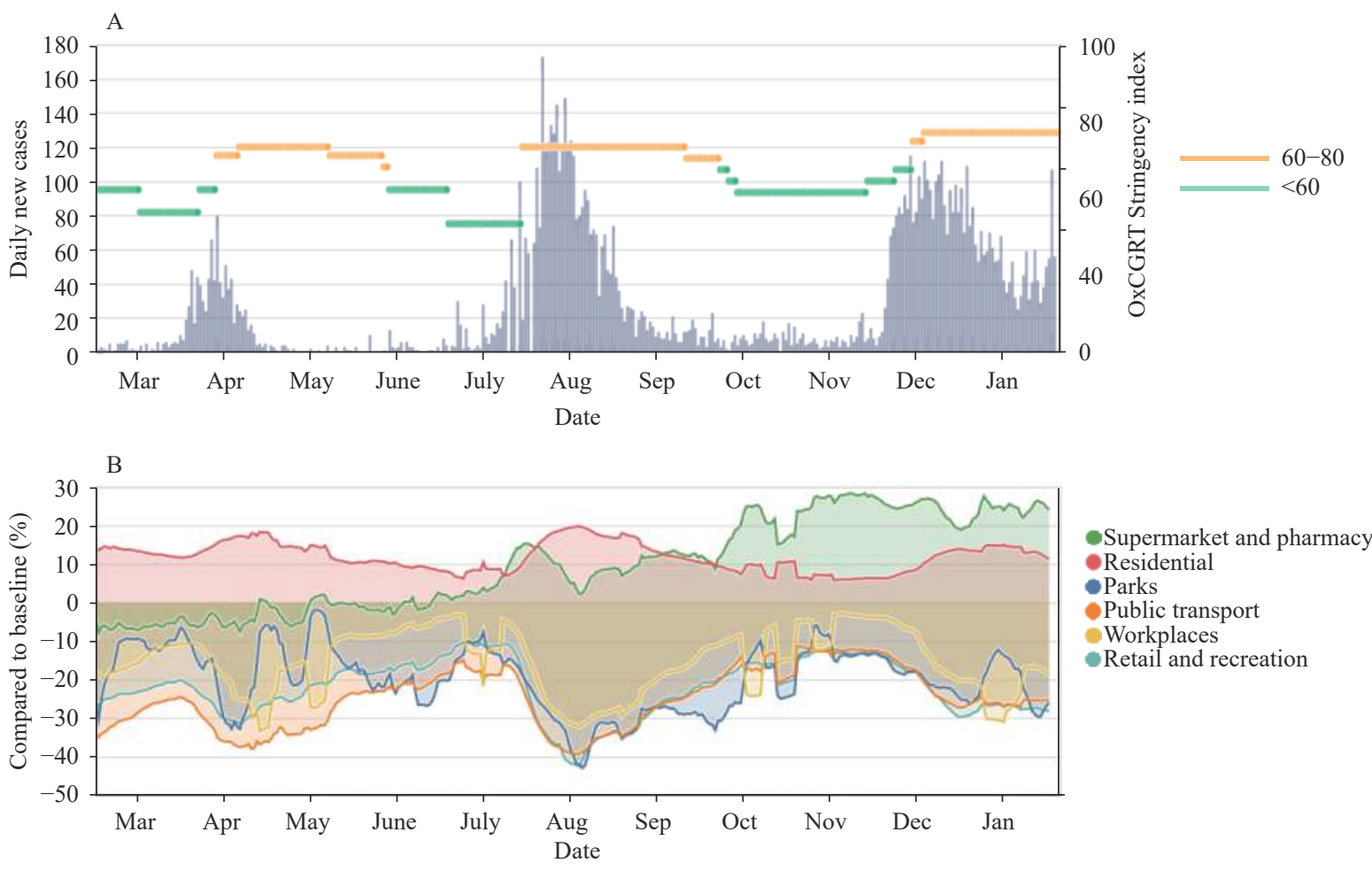

FIGURE 1. Description of the COVID-19 epidemic in Hong Kong from February 15, 2020 to January 20, 2021. (A) Daily new cases and the oxford COVID-19 government response tracker (OxCGRT). (B) Google COVID-19 community mobility (7-day moving average) compared to baseline(\%).

Notes: In Figure 1A, the yellow horizontal bars represent an OxCGRT index of 60-80 and the green bars indicate a value below 60 , which is relatively loose. In general, as the number of daily new cases increases, the government will respond by tightening control measures (OxCGRT increases), resulting in decreases in daily new cases.

From the outbreak in December 2020, the policy is very strict, four indicators such as parks, retail and recreation, public transport and workplaces were decreased but not as good as the outbreak in August, and people's frequency of going out to supermarket and pharmacy is still at a relatively high level, which led to the long duration of this epidemic. Since OxGRT index doesn't change frequently, the correlation analysis between daily new cases and Google Mobility Trends is taken in order to assess the impact of population mobility on epidemic.

Due to the lag between the changes in mobility and the number of daily new cases, rank correlation analysis was used to obtain the number of lag days and correlation coefficients with the strongest correlation between each indicator and the number of daily new cases (G). Among the six indicators of Google Mobility Trends, residential mobility being positively correlated with daily new cases indicated a high degree of compliance, where as the other five indicators being negatively correlated with daily new cases indicated a high degree of compliance. All statistical analyses were conducted by $\mathrm{R}$ statistical software(version 4.0.3; The
R Foundation for Statistical Computing, Vienna, Austria).

The results of rank correlation analysis showed that the lag days with the strongest correlation between the 6 indicators and the number of new cases per day ranged from 1 to 14 days. The supermarket and pharmacy category was positively correlated with the number of new cases per day, $r=0.351(P<0.001)$. The residential category was positively correlated with the number of new cases per day, $r=0.487(P<0.001)$. The other categories of retail and recreation, workplaces, parks, and public transport were negatively correlated with the number of new cases per day, the $r$ values were $-0.620,-0.604,-0.544$, and -0.392 , respectively, and $P$ values were all less than 0.001 . Among them, retail and recreation and workplaces were strongly correlated with the number of new cases per day; they both were higher than 0.6 (Table 1). Statistical significance was attributed to $\mathrm{p}$ values $(P<0.001)$.

\section{DISCUSSION}

The speed of the epidemic may be related to many 
TABLE 1. The lag days and correlation analysis of the six indicators of Google Mobility Trends relative to the number of daily new cases of COVID-19.

\begin{tabular}{lccc}
\hline $\begin{array}{c}\text { The categories of Google } \\
\text { Mobility Trends }\end{array}$ & Lag days & $\boldsymbol{r}$ & $\boldsymbol{P}$ \\
\hline Supermarket and pharmacy & 1 & 0.351 & $<0.001$ \\
Residential & 14 & 0.487 & $<0.001$ \\
Retail and recreation & 14 & -0.620 & $<0.001$ \\
Workplace & 14 & -0.604 & $<0.001$ \\
Park & 11 & -0.544 & $<0.001$ \\
Public transport & 14 & -0.392 & $<0.001$ \\
\hline
\end{tabular}

factors, such as virus variants, the number of super spreaders, etc. However, mobility restrictions and control measures can provide important information for the possibility of accelerated transmission. Analysis of daily new cases, Hong Kong Mobility Trends, and OxCGRT found that when the number of new cases increased, the government promptly tightened the response, and the Google Mobility Trends reflected that when residents had a high degree of compliance, the epidemic was well controlled. Delays in policy implementation and compliance will make the epidemic much harder to control. Overall, the population's compliance with the control policy in Hong Kong is very good in the whole year.

The last COVID-19 epidemic in Hong Kong is still going on and rebound slightly, which may be due to higher mobility comparing with the previous epidemic. Based on this situation, more protection in public, expanding testing and strict contact tracing still needs to be strengthened. Social distancing policies need to be strictly implemented among citizens, especially mask wearing and restrictions on gatherings.

Government should evaluate the six indicators of the Google Mobility Trends, especially supermarket and pharmacy, to isolate the purpose behind mobility of residents and to propose targeted solutions. Combing the relationship between the daily number of new confirmed cases, changes in mobility, and the OxCGRT for comparative analysis, researchers can predict the trends of the epidemic, choose proper times and intensities for tightening policies, and collect evidence to more precisely control the epidemic in the future. The results of the correlation analysis of daily new cases and changes in mobility concluded that the two categories of retail and recreation and workplaces were strongly correlated with the number of daily new cases, which indicates that these two venue categories should be targeted in effective epidemic control.

Although Hong Kong has relatively ample medical resources, significant increases in critical and severe cases will put tremendous pressure on the finite capacity of hospital beds. Based on the high population density, it is recommended that the government establish a list of household material reserves; regulate certain types of emergency supplies, emergency tools, and emergency medicines; and carry out targeted expansion of reserves.

Virus variants also became prevalent in some countries in mid-December 2020. In the meanwhile, the government has approved a COVID-19 vaccine that meets safety and efficacy for emergency use and has ordered 22.5 million doses. Strict testing should be continued for international students returning to Hong Kong to prevent the import of virus variants, strengthen quarantine hotel management, and avoid cross-infection.

This article didn't take into account of the virus mutation, climate factors which may also lead to the rapid increase of the cases. The OxCGRT Stringency Index of control measures were not integrated into the correlated model directly, which was lack of the assessment of control policy.

Acknowledgements: The authors would like to thank Yidu Cloud (Beijing) Technology Co. Ltd. for assistance with COVID-19 related data collection.

Conflicts of Interest: No conflicts of interest were reported.

doi: $10.46234 / \mathrm{ccdcw} 2021.020$

\# Corresponding author: Xiaopeng Qi, qixp@chinacdc.cn.

${ }^{1}$ Chinese Center for Disease Control and Prevention, Beijing, China.

Submitted: January 08, 2021; Accepted: January 22, 2021

\section{REFERENCES}

1. The Government of the Hong Kong Special Administrative Region. Latest local situation of COVID-19. https://www.coronavirus.gov.hk/ eng/index.html\#What_is_COVID-19. [2021-1-20].

2. The Government of the Hong Kong Special Administrative Region. Statistics on testing for COVID-19 in Hong Kong. https://www. coronavirus.gov.hk/eng/index.html\#What_is_COVID-19. [2021-1-20].

3. The Government of the Hong Kong Special Administrative Region. Situation on public hospitals and confirmed cases. https://www.coro navirus.gov.hk/eng/index.html\#What_is_COVID-19. [2020-12-24].

4. Google. COVID-19 Community Mobility Reports. https://www.google.com/covid19/mobility/. [2021-01-20].

5. Oxford University. COVID-19 Government Response Tracker (OxCGRT). [2021-01-21]. https://www.bsg.ox.ac.uk/research/researchprojects/coronavirus-government-response-tracker.

6. Chan BKC. Data analysis using R programming. In: Biostatistics for human genetic epidemiology. Cham: Springer. 2018. http://dx.doi.org/ 10.1007/978-3-319-93791-5_2. 